\title{
A new characterization between osculating strip curves and ruled surfaces in Lorentzian space
}

\author{
Suha Yilmaz ${ }^{1}$ and Yasin Unluturk ${ }^{2}$ \\ ${ }^{1}$ Buca Faculty of Education, Dokuz Eylul University, Buca-Izmir, Turkey \\ ${ }^{2}$ Department of Mathematics, Kirklareli University, Kirklareli, Turkey
}

Received: 15 February 2016, Accepted: 30 September 2016

Published online: 24 October 2016.

\begin{abstract}
In this work, we study the conditions between osculating strip curves and ruled surfaces in Lorentzian space. For this study, we establish a system of differential equations characterizing both spacelike and timelike ruled surfaces in Lorentzian space by using the invariant quantities of osculating strip curves on the given ruled surfaces. We obtain the solutions of these systems for special cases. Regarding to these special solutions, we give some results of relations between osculating strip curves and ruled surfaces in Lorentzian space.
\end{abstract}

Keywords: Lorentz space, osculating strip curve, normal curve,Blaschke frame, Darboux frame.

\section{Introduction}

The theory of Klein geometry is of invariants of a transitive transformation group. The structure of the underlying Lie group is an essential element in geometry. This fact can be nicely seen in the theory of ruled surfaces. The first studies from this perspective were made by W. Blaschke and E. Study. The main idea is to replace points by lines as fundamental building blocks of geometric beings [6].

The relations between curves and ruled surfaces were first given by Blaschke in [3]. Blaschke established this relation by means of Study theorem which works in dual space. By Study map, ruled surfaces are considered as spherical curves in dual space. The conditions between curves and ruled surfaces were studied by establishing a system of differential equations which determined ruled surfaces in Euclidean space in [7].

Based on the thought "curve-surface strip", the conditions between geodesic curves and ruled surfaces were dealt with by Pekmen [10] by means of Blaschke invariants in [3]. Later, Şişman wrote an MA dissertation about this problem. He tried to solve the system of differential equations determining ruled surfaces in dual Euclidean space by using the invariants of the surface strip curves, he couldn't obtain the general solution of this system. But he had the solutions by provided that the surface strip curves were osculating, curvature and geodesic strip curves [12]. After Şişman's MA thesis, Pasinli took the subject into consideration by not using the vanishing of Darboux invariants [9]. Also, Ayyldız studied the conditions between curves and semi-ruled surfaces in Lorentz 3-space [2]. Then the conditions between geodesic curves and ruled surfaces were studied in Lorentzian space by Ayylldı [1]. Some interesting results between curves and ruled surfaces were also obtained in dual Lorentzian space in $[4,5]$.

In this work, we study the conditions between osculating strip curves and ruled surfaces in dual Lorentz space $\mathbb{D}_{1}^{3}$. For

\footnotetext{
*Corresponding author e-mail: yasinunluturk@klu.edu.tr
} 
this study, we establish a system of differential equations characterizing both spacelike and timelike ruled surfaces in dual Lorentz space $\mathbb{D}_{1}^{3}$ by using the invariant quantities of osculating strip curves on the given ruled surfaces. We obtain the solutions of these systems for special cases. Regarding to these special solutions, we give some results of relations between osculating strip curves and ruled surfaces in dual Lorentz space $\mathbb{D}_{1}^{3}$.

\section{Preliminaries}

Let $\mathbb{R}_{1}^{3}$ be the three-dimensional Minkowski space, that is, the three-dimensional real vector space $\mathbb{R}^{3}$ with the metric

$$
\langle d x, d x\rangle=d x_{1}^{2}+d x_{2}^{2}-d x_{3}^{2},
$$

where $\left(x_{1}, x_{2}, x_{3}\right)$ denotes the canonical coordinates in $R^{3}$. An arbitrary vector $x$ of $E_{1}^{3}$ is said to be spacelike if $\langle x, x\rangle>0$ or $x=0$, timelike if $\langle x, x\rangle<0$ and lightlike or null if $\langle x, x\rangle=0$ and $x \neq 0$. A timelike or light-like vector in $E_{1}^{3}$ is said to be causal. For $x \in E_{1}^{3}$ the norm is defined by $\|x\|=\sqrt{|\langle x, x\rangle|}$, then the vector $x$ is called a spacelike unit vector if $\langle x, x\rangle=1$ and a timelike unit vector if $\langle x, x\rangle=-1$. Similarly, a regular curve in $E_{1}^{3}$ can locally be spacelike, timelike or null (lightlike), if all of its velocity vectors are spacelike, timelike or null (lightlike), respectively [8].

Dual numbers are given by the set

$$
\mathbb{D}=\left\{\widehat{x}=x+\xi x^{*} ; x, x^{*} \in \mathbb{R}\right\},
$$

where the symbol $\xi$ designates the dual unit with the property $\xi^{2}=0$ for $\xi \neq 0$. Dual angle is defined as $\widehat{\theta}=\theta+\xi \theta^{*}$, where $\theta$ is the projected angle between two spears and $\theta^{*}$ is the shortest distance between them. The set $\mathbb{D}$ of dual numbers is a commutative ring the the operations + and $\cdot$. The set

$$
\mathbb{D}^{3}=\mathbb{D} \times \mathbb{D} \times \mathbb{D}=\left\{\widehat{\varphi}=\varphi+\xi \varphi^{*} ; \varphi, \varphi^{*} \in \mathbb{E}^{3}\right\}
$$

is a module over the ring $\mathbb{D}[15]$.

For any $\widehat{a}=a+\xi a^{*}, \widehat{b}=b+\xi b^{*} \in \mathbb{D}^{3}$, the Lorentzian inner product of $\widehat{a}$ and $\widehat{b}$ is defined by

$$
<\widehat{a}, \widehat{b}>=<a, b>+\xi\left(<a^{*}, b>+<a, b^{*}>\right)
$$

The dual space $\mathbb{D}^{3}$ together with this Lorentzian inner product is called the dual Lorentzian space and is denoted by $\mathbb{D}_{1}^{3}$ [14]. For $\widehat{\varphi} \neq 0$, the norm $\|\widehat{\varphi}\|$ of $\widehat{\varphi}$ is defined by

$$
\|\widehat{\varphi}\|=\sqrt{<\widehat{\varphi}, \widehat{\varphi}\rangle}
$$

A dual vector $\widehat{\omega}=\omega+\xi \omega^{*}$ is called dual spacelike vector if $\langle\widehat{\omega}, \widehat{\omega}\rangle>0$ or $\widehat{\omega}=0$, dual timelike vector if $\langle\widehat{\omega}, \widehat{\omega}\rangle<0$ and dual null (lightlike) vector if $\langle\widehat{\omega}, \widehat{\omega}\rangle=0$ for $\widehat{\omega} \neq 0$. Therefore, an arbitrary dual curve which is a differential mapping onto $\mathbb{D}_{1}^{3}$ can locally be dual spacelike, dual timelike or dual null if its velocity vector is dual spacelike, dual timelike or dual null, respectively. Also, for the dual vectors $\widehat{a}, \widehat{b} \in \mathbb{D}_{1}^{3}$, Lorentzian vector product of these dual vectors is defined by

$$
\widehat{a} \times \widehat{b}=a \times b+\xi\left(a^{*} \times b+a \times b^{*}\right)
$$

where $a \times b$ is the classical cross product according to the signature $(+,+,-)$ [14]. 
In $\mathbb{D}_{1}^{3}$, the dual Lorentzian unit sphere is given as

$$
S_{1}^{2}=\left\{\widehat{\varphi}=\varphi+\xi \varphi^{*} ;\|\widehat{\varphi}\|=(1,0) ; \varphi, \varphi^{*} \in \mathbb{R}_{1}^{3}, \varphi \text { spacelike }\right\}
$$

and the dual hyperbolic unit sphere is defined as

$$
H_{0}^{2}=\left\{\widehat{\varphi}=\varphi+\xi \varphi^{*} ;\|\widehat{\varphi}\|=(1,0) ; \varphi, \varphi^{*} \in \mathbb{R}_{1}^{3}, \varphi \text { timelike }\right\} .
$$

The oriented timelike and spacelike lines in $\mathbb{E}_{1}^{3}$ are presented by timelike and spacelike unit vectors with three components in $\mathbb{D}_{1}^{3}$, respectively. A differentiable curve on $H_{0}^{2}$ corresponds to a timelike ruled surface while a differentiable curve on $S_{1}^{2}$ corresponds to any ruled surface [14].

Theorem 1. (E. Study) The oriented lines in $R^{3}$ are in one-to-one correspondence with the points of the dual unit sphere $A . A=1$ in $D^{3}[11]$.

A differentiable curve $\mathrm{A}(\mathrm{u})$ on the dual unit sphere, depending on a real unit parameter $\mathrm{u}$, represents a differentiable family of straight lines in $\mathrm{R}^{3}$ : a ruled surface. The lines $\mathrm{A}(\mathrm{u})$ are the generators or rulings of the surface.

The distribution parameter of the ruled surface determined by

$$
X(t)=x(t)+\xi x^{*}(t)
$$

is defined by

$$
\delta=\frac{\left\langle x^{\prime}(t), x^{* \prime}(t)\right\rangle}{\left\langle x^{\prime}(t), x^{\prime}(t)\right\rangle}=\frac{p^{*}}{p} .
$$

If $\delta=0, p^{*}=0$ then the ruled surface is a developable surface [3].

Let a curve $\alpha(s)$ on a surface $M$ in Lorentz space be given by the arc-length parameter. The Frenet trihedron which belongs to this curve differs as follows:

The Frenet formulae of a spacelike curve with timelike principal normal are given as

$$
\left[\begin{array}{l}
T^{\prime} \\
N^{\prime} \\
B^{\prime}
\end{array}\right]=\left[\begin{array}{lll}
0 & \kappa & 0 \\
\kappa & 0 & \tau \\
0 & \tau & 0
\end{array}\right]\left[\begin{array}{l}
T \\
N \\
B
\end{array}\right],
$$

where $\langle T, T\rangle=1,\langle N, N\rangle=-1,\langle B, B\rangle=1,\langle T, N\rangle=\langle T, B\rangle=\langle N, B\rangle=0$ and $\kappa$ and $\tau$ are curvature and torsion of the spacelike curve, respectively [14].

The Frenet formulae of a timelike curve are given as

$$
\left[\begin{array}{c}
T^{\prime} \\
N^{\prime} \\
B^{\prime}
\end{array}\right]=\left[\begin{array}{ccc}
0 & \kappa & 0 \\
\kappa & 0 & \tau \\
0 & -\tau & 0
\end{array}\right]\left[\begin{array}{c}
T \\
N \\
B
\end{array}\right],
$$

where $\langle T, T\rangle=-1,\langle N, N\rangle=1,\langle B, B\rangle=1,\langle T, N\rangle=\langle T, B\rangle=\langle N, B\rangle=0$ and $\kappa$ and $\tau$ are curvature and torsion of the spacelike curve, respectively [14]. 
According to Lorentzian causal characters of the surface $S$ and the curve $\alpha(s)$ on $S$, the Darboux derivative formulae are given as follows:

(i) If both the curve and surface are spacelike ones, then the formulae are

$$
\left[\begin{array}{l}
\eta_{1}^{\prime}(s) \\
\eta_{2}^{\prime}(s) \\
\eta_{3}^{\prime}(s)
\end{array}\right]=\left[\begin{array}{ccc}
0 & \rho_{g} & \rho_{n} \\
-\rho_{g} & 0 & \tau_{g} \\
\rho_{n} & \tau_{g} & 0
\end{array}\right]\left[\begin{array}{l}
\eta_{1}(s) \\
\eta_{2}(s) \\
\eta_{3}(s)
\end{array}\right]
$$

(ii) If both the curve and surface are timelike ones, then the formulae are

$$
\left[\begin{array}{l}
\eta_{1}^{\prime}(s) \\
\eta_{2}^{\prime}(s) \\
\eta_{3}^{\prime}(s)
\end{array}\right]=\left[\begin{array}{ccc}
0 & \rho_{g} & \rho_{n} \\
\rho_{g} & 0 & -\tau_{g} \\
\rho_{n} & \tau_{g} & 0
\end{array}\right]\left[\begin{array}{l}
\eta_{1}(s) \\
\eta_{2}(s) \\
\eta_{3}(s)
\end{array}\right]
$$

In these formulae, $\rho_{g}, \rho_{n}$ and $\tau_{g}$ are called the geodesic curvature, the normal curvature, and the geodesic torsion, respectively [14].

A ruled surface in dual space is represented by the following dual unit vectorial fuction

$$
\vec{X}(t)=x(t)+\xi x^{*}(t)
$$

where $\xi=(0,1)$ is the dual unit. Let the vectors

$$
\begin{aligned}
& X_{1}(t)=x_{1}(t)+\xi x_{1}^{*}(t), \\
& X_{2}(t)=\frac{X_{1}^{\prime}(t)}{\left\|X_{1}^{\prime}(t)\right\|}=\frac{X_{1}^{\prime}(t)}{P}, \\
& X_{3}(t)=X_{1}(t) \times X_{2}(t)
\end{aligned}
$$

be a trihedron depending on the ruled surface in $\mathbb{D}_{1}^{3}$. This trihedron is called as Blaschke trihedron. It differs according to the causal character of ruled surface as follows:

(i) If the ruled surface is a spacelike surface, then the Blaschke derivative formulae are

$$
\left[\begin{array}{l}
X_{1}^{\prime}(t) \\
X_{2}^{\prime}(t) \\
X_{3}^{\prime}(t)
\end{array}\right]=\left[\begin{array}{ccc}
0 & P & 0 \\
-P & 0 & Q \\
0 & Q & 0
\end{array}\right]\left[\begin{array}{l}
X_{1}(t) \\
X_{2}(t) \\
X_{3}(t)
\end{array}\right],
$$

(ii) If the ruled surface is a timelike surface, then the Blaschke derivative formulae are

$$
\left[\begin{array}{l}
X_{1}^{\prime}(t) \\
X_{2}^{\prime}(t) \\
X_{3}^{\prime}(t)
\end{array}\right]=\left[\begin{array}{ccc}
0 & P & 0 \\
P & 0 & Q \\
0 & -Q & 0
\end{array}\right]\left[\begin{array}{l}
X_{1}(t) \\
X_{2}(t) \\
X_{3}(t)
\end{array}\right] .
$$

In equations (8), (9), the dual invariants of ruled surfaces are

$$
P=p(t)+\xi p^{*}(t), Q=q(t)+\xi q^{*}(t)
$$

where $P=\left\|X_{1}^{\prime}(t)\right\|, Q=\left\langle X_{2}^{\prime}(t), X_{3}(t)\right\rangle[1]$

Let $S$ and $\alpha$ be a surface and a curve, respectively, in $E_{1}^{3}$. The points of the curve $\alpha$ and the tangent vectors of the surface 
$S$ at the same points which constitutes a geometric being are called as strip [13].

Strips which satisfy the condition $\rho_{n}=\left\langle\eta_{1}^{\prime}, \eta_{3}\right\rangle=0$ are called osculating strips [3].

If the moments of the Darboux vector at the point $\alpha(s)$ are taken with respect to origin in the coordinate system $(O ; x, y, z)$, the dual unit vectors which are defined as

$$
X_{i}(s)=\eta_{i}(s)+\xi \eta_{i}^{*}(s)=\eta_{i}(s)+\xi\left(\alpha(s) \times \eta_{i}(s)\right), 1 \leq i, j \leq 3
$$

form the base $\left\{X_{1}(s), X_{2}(s), X_{3}(s)\right\}$ in dual Lorentz space and these vectors have the following property

$$
\left\langle X_{i}(s), X_{j}(s)\right\rangle=\left\{\begin{array}{l}
\varepsilon\left(X_{i}\right) \text { if } i=j \\
0 \text { if } i \neq j .
\end{array}\right.
$$

When the point $\alpha(s)$ traces the curve $\alpha$ in Lorentz space, the dual unit vector $X_{1}(s)$ generates the surface in dual Lorentz space. The frame

$$
\left\{X_{1}(s), X_{2}(s), X_{3}(s)\right\}
$$

belonging to the generator $X_{1}(s)$ is the Blaschke trihedron of the surface.

\section{The characterizations between spacelike osculating strip curves and spacelike ruled surfaces} in $\mathbb{D}_{1}^{3}$

In this condition, we choose both the curve $\alpha$ and the ruled surface $X_{1}$ as a spacelike curve and a spacelike surface, respectively. The vector $\alpha(s)$ is written with respect to Darboux vector is

$$
\alpha(s)=m(s) \eta_{1}(s)+n(s) \eta_{2}(s)+k(s) \eta_{3}(s)
$$

where $m=m(s), n=n(s), k=k(s)$.

Hence we obtain the following relations

$$
\begin{aligned}
& X_{1}(s)=\eta_{1}(s)+\xi\left[-k(s) \eta_{2}(s)-n(s) \eta_{3}(s)\right] \\
& X_{2}(s)=\eta_{2}(s)+\xi\left[k(s) \eta_{1}(s)+m(s) \eta_{3}(s)\right] \\
& X_{3}(s)=\eta_{3}(s)+\xi\left[-n(s) \eta_{1}(s)+m(s) \eta_{2}(s)\right]
\end{aligned}
$$

If the coefficients $m(s), n(s), k(s)$ are found, then the spacelike ruled surface $X_{1}(s)$ can be determined with respect to the invariants of the curve $\alpha(s)$. Differentiating (11) with respect to $s$, and then substituting (4) in it gives

$$
\left\{\begin{array}{l}
m^{\prime}(s)+\rho_{g} n(s)+\rho_{n} k(s)=1 \\
m(s) \rho_{g}+n^{\prime}(s)+k(s) \tau_{g}=0 \\
m(s) \rho_{n}+n(s) \tau_{g}+k^{\prime}(s)=0
\end{array}\right.
$$

Since the condition for the curve $\alpha$ to be an osculating strip curve, that is $\rho_{n}=0$, the equation (13) becomes

$$
\left\{\begin{array}{l}
m^{\prime}(s)+\rho_{g} n(s)=1 \\
m(s) \rho_{g}+n^{\prime}(s)+k(s) \tau_{g}=0 \\
n(s) \tau_{g}+k^{\prime}(s)=0
\end{array}\right.
$$


We will study the sytem of differential equations (14) for certain special cases as follows:

Case 1. If $m(s)=0$, the curve $\alpha$ is located in affine subspace combined with the vector space $S_{p}\left\{\eta_{2}(s), \eta_{3}(s)\right\}$ at the point $\alpha(s)$, then the system (14) turns into

$$
\left\{\begin{array}{l}
\rho_{g} n(s)=1 \\
n^{\prime}(s)+k(s) \tau_{g}=0 \\
n(s) \tau_{g}+k^{\prime}(s)=0
\end{array}\right.
$$

From $(15)_{2}$ and $(15)_{3}$, we have

$$
k^{\prime \prime}(s)-\frac{\tau_{g}^{\prime}}{\tau_{g}} k^{\prime}(s)-\tau_{g}^{2} k(s)=0 .
$$

If we change the parameter as $t=\int_{0}^{s} \tau_{g} d s$, we obtain $\frac{d^{2} k}{d t^{2}}-k=0$. Thus the solution of $k$ is as follows:

$$
k=c_{1} e^{\left(\int_{0}^{s} \tau_{g} d s\right)}+c_{2} e^{-\left(\int_{0}^{s} \tau_{g} d s\right)}
$$

where $c_{1}$ and $c_{2}$ are real constants. Also from $(15)_{1}$, it is clear that

$$
n(s)=\frac{1}{\rho_{g}} .
$$

Rewriting (17) and (18) in (11), we obtain the curve $\alpha$ as

$$
\alpha(s)=\frac{1}{\rho_{g}} \eta_{2}(s)+\left[c_{1} e^{\left(\int_{0}^{s} \tau_{g} d s\right)}+c_{2} e^{-\left(\int \tau_{0}^{s} \tau_{g} d s\right)}\right] \eta_{3}(s) .
$$

Thereby, Blaschke vectors of the spacelike ruled surface $X_{1}(s)$ are determined by the invariants of Darboux trihedron as follows:

$$
\begin{aligned}
& X_{1}(s)=\eta_{1}(s)+\xi\left[-\left[c_{1} e^{\left(\int \tau_{g} \tau_{g} d s\right)}+c_{2} e^{-\left(\int \tau_{g} d s\right)}\right] \eta_{2}(s)-\frac{1}{\rho_{g}} \eta_{3}(s)\right], \\
& X_{2}(s)=\eta_{2}(s)+\xi\left[c_{1} e^{\left(\int \tau_{g} d s\right)}+c_{2} e^{-\left(\int \tau_{g} d s\right)}\right] \eta_{1}(s), \\
& X_{3}(s)=\eta_{3}(s)+\xi\left[-\frac{1}{\rho_{g}} \eta_{1}(s)\right] .
\end{aligned}
$$

Thus we can give the following result.

Corollary 1. The curve $\alpha$ is determined by (19) and the Blaschke vectors of spacelike ruled surface $X_{1}(s)$ are determined by the invariants of Darboux trihedron as in (20).

If $c_{1}=c_{2}=0$, the equation (20) becomes

$$
\begin{aligned}
& X_{1}(s)=\eta_{1}(s)+\xi\left[-\frac{1}{\rho_{g}} \eta_{3}(s)\right], \\
& X_{2}(s)=\eta_{2}(s), \\
& X_{3}(s)=\eta_{3}(s)+\xi\left[-\frac{1}{\rho_{g}} \eta_{1}(s)\right] .
\end{aligned}
$$


Differentiating $(21)_{1}$ and $(21)_{3}$ with respect to $s$, and then using (8), we have

$$
p=\rho_{g}, p^{*}=-\frac{\tau_{g}}{\rho_{g}}, q=\tau_{g}, q^{*}=1
$$

Corollary 2. The real and dual parts of the dual invariants of spacelike ruled surface $X_{1}$ are obtained as in (22).

Case 2. If $n(s)=0$, the curve $\alpha$ is located in affine subspace combined with the vector space $S_{p}\left\{\eta_{1}(s), \eta_{3}(s)\right\}$ at the point $\alpha(s)$, hence the system (14) turns into

$$
\left\{\begin{array}{l}
m^{\prime}(s)=1 \\
m(s) \rho_{g}+k(s) \tau_{g}=0 \\
k^{\prime}(s)=0
\end{array}\right.
$$

From $(23)_{1}$ and $(23)_{3}$, we have

$$
m=s+c_{1}, k=c_{2}
$$

and also from $(23)_{2}$, we obtain a ratio between the geodesic torsion and the geodesic curvature as

$$
\frac{\rho_{g}}{\tau_{g}}=-\frac{c_{2}}{s+c_{1}}
$$

Rewriting (24) in (11), we obtain the curve $\alpha$ as

$$
\alpha(s)=\left(s+c_{1}\right) \eta_{1}(s)+c_{2} \eta_{3}(s)
$$

Thereby, Blaschke vectors of the spacelike ruled surface $X_{1}(s)$ are determined by the invariants of Darboux trihedron as follows:

$$
\begin{aligned}
& X_{1}(s)=\eta_{1}(s)+\xi\left[-c_{2} \eta_{2}(s)\right], \\
& X_{2}(s)=\eta_{2}(s)+\xi\left[c_{2} \eta_{1}(s)+\left(s+c_{1}\right) \eta_{3}(s)\right], \\
& X_{3}(s)=\eta_{3}(s)+\xi\left[\left(s+c_{1}\right) \eta_{2}(s)\right] .
\end{aligned}
$$

Thus we can give the following result:

Corollary 3. The curve $\alpha$ is determined by (25) and the Blaschke vectors of spacelike ruled surface $X_{1}(s)$ are determined by the invariants of Darboux trihedron as in (26).

If $c_{1}=c_{2}=0$, the equation (20) becomes

$$
\begin{aligned}
& X_{1}(s)=\eta_{1}(s) \\
& X_{2}(s)=\eta_{2}(s)+\xi\left(s \eta_{3}(s)\right), \\
& X_{3}(s)=\eta_{3}(s)+\xi\left(s \eta_{2}(s)\right) .
\end{aligned}
$$

Differentiating $(27)_{1}$ and $(27)_{3}$ with respect to $s$, and then using (8), we have

$$
p=\rho_{g}, p^{*}=0, q=\tau_{g}, q^{*}=1
$$

Thus we can give the following result.

Corollary 4. The real and dual parts of the dual invariants of spacelike ruled surface $X_{1}$ are obtained as in (28).

From (28) and (1), we get the following result. 
Corollary 5. If $c_{1}=c_{2}=0$, then the spacelike ruled surface generated by the line $X_{1}$ is a developable surface.

Case 3. If $k(s)=0$, the curve $\alpha$ is located in affine subspace combined with the vector space $S_{p}\left\{\eta_{1}(s), \eta_{2}(s)\right\}$ at the point $\alpha(s)$, then the system (14) turns into

$$
\left\{\begin{array}{l}
m^{\prime}(s)+\rho_{g} n(s)=1 \\
m(s) \rho_{g}+n^{\prime}(s)=0 \\
n(s) \tau_{g}=0
\end{array}\right.
$$

From (29) $)_{1}$ and $(29)_{3}$, we have

$$
m^{\prime \prime}(s)+\frac{\rho_{g}^{\prime}}{\rho_{g}}\left(1-m^{\prime}(s)\right)-\rho_{g}^{2} m(s)=0
$$

If we change the parameter as $t=\int_{0}^{s} \rho_{g} d s$ in (30), we obtain

$$
\frac{d^{2} m}{d t^{2}}-m=\frac{\rho_{g}^{\prime}}{\rho_{g}^{3}}
$$

Thus the solution of $m$ is as follows:

$$
m=c_{1} e^{\left(\iint_{0}^{s} \rho_{g} d s\right)}+c_{2} e^{\left(-\int_{0}^{s} \rho_{g} d s\right)}+\frac{1}{D^{2}-1}\left(-\frac{\dot{\rho_{g}}}{\rho_{g}^{3}}\right)
$$

where $c_{1}$ and $c_{2}$ are real constants. Also from $(29)_{3}$, it is clear that

$$
n(s)=0
$$

Rewriting (31) and (32) in (11), we obtain the curve $\alpha$ as

$$
\alpha(s)=\left[c_{1} e^{\left(\int \rho_{0} \rho_{g} d s\right)}+c_{2} e^{\left(-\int_{0}^{s} \rho_{g} d s\right)}+\frac{1}{D^{2}-1}\left(-\frac{\dot{\rho_{g}}}{\rho_{g}^{3}}\right)\right] \eta_{1}(s),
$$

where $c_{1}, c_{2} \in R, \dot{\rho_{g}}=\frac{d \rho_{g}}{d t}, D=\frac{d}{d t}$.

Thereby, Blaschke vectors of the spacelike ruled surface $X_{1}(s)$ are determined by the invariants of Darboux trihedron as follows:

$$
\begin{aligned}
& X_{1}(s)=\eta_{1}(s), \\
& X_{2}(s)=\eta_{2}(s)+\xi\left[c_{1} e^{\left(\int \rho_{0} d s\right)}+c_{2} e^{\left(-\int_{0}^{s} \rho_{g} d s\right)}+\frac{1}{D^{2}-1}\left(-\frac{\dot{\rho_{g}}}{\rho_{g}^{3}}\right)\right] \eta_{3}(s), \\
& X_{3}(s)=\eta_{3}(s)+\xi\left[c_{1} e^{\left(\int_{0}^{s} \rho_{g} d s\right)}+c_{2} e^{\left(-\int_{0}^{s} \rho_{g} d s\right)}+\frac{1}{D^{2}-1}\left(-\frac{\dot{\rho}_{g}}{\rho_{g}^{3}}\right)\right] \eta_{2}(s) .
\end{aligned}
$$

Thus we can give the following result.

Corollary 6. The curve $\alpha$ is determined by (33) and the Blaschke vectors of spacelike ruled surface $X_{1}(s)$ are determined by the invariants of Darboux trihedron as in (34). 
If $c_{1}=c_{2}=0$, the equation (34) becomes

$$
\begin{aligned}
& X_{1}(s)=\eta_{1}(s), \\
& X_{2}(s)=\eta_{2}(s)+\xi\left[\frac{1}{D^{2}}\left(\frac{\rho_{g}^{\prime}}{\rho_{g}}\right)\right] \eta_{3}(s), \\
& X_{3}(s)=\eta_{3}(s)+\xi\left[\frac{1}{D^{2}}\left(\frac{\rho_{g}^{\prime}}{\rho_{g}}\right)\right] \eta_{2}(s) .
\end{aligned}
$$

Differentiating (35) $)_{1}$ and $(35)_{3}$ with respect to $s$, and then using (8), we have

$$
p=\rho_{g}, p^{*}=0, q=\tau_{g}, q^{*}=0 .
$$

Thus we can give the following result.

Corollary 7. The real and dual parts of the dual invariants of spacelike ruled surface $X_{1}$ are obtained as in (22).

From (36) and (1), we get the following result.

Corollary 8. If $c_{1}=c_{2}=0$, then the spacelike ruled surface generated by the line $X_{1}$ is a developable surface.

\section{The characterizations between timelike osculating strip curves and timelike ruled surfaces in $\mathbb{D}_{1}^{3}$}

In this condition, we choose both the curve $\alpha$ and the ruled surface $X_{1}$ as a timelike curve and a timelike surface, respectively. The vector $\alpha(s)$ is written with respect to Darboux vector as

$$
\alpha(s)=m(s) \eta_{1}(s)+n(s) \eta_{2}(s)+k(s) \eta_{3}(s),
$$

where $m=m(s), n=n(s), k=k(s)$.

Hence we obtain the following relations

$$
\begin{aligned}
& X_{1}(s)=\eta_{1}(s)+\xi\left[-k(s) \eta_{2}(s)+n(s) \eta_{3}(s)\right], \\
& X_{2}(s)=\eta_{2}(s)+\xi\left[-k(s) \eta_{1}(s)+m(s) \eta_{3}(s)\right], \\
& X_{3}(s)=\eta_{3}(s)+\xi\left[n(s) \eta_{1}(s)+m(s) \eta_{2}(s)\right] .
\end{aligned}
$$

If the coefficients $m(s), n(s), k(s)$ are found, then the timelike ruled surface $X_{1}(s)$ can be determined with respect to the invariants of the curve $\alpha(s)$. Differentiating (37) with respect to $s$, and then substituting (5) in it gives

$$
\left\{\begin{array}{l}
m^{\prime}(s)+\rho_{g} n(s)+\rho_{n} k(s)=1 \\
m(s) \rho_{g}+n^{\prime}(s)+k(s) \tau_{g}=0 \\
m(s) \rho_{n}-n(s) \tau_{g}+k^{\prime}(s)=0
\end{array}\right.
$$

Since the condition for the curve $\alpha$ to be a osculating strip curve, that is $\rho_{n}=0$, the equation (39) becomes

$$
\left\{\begin{array}{l}
m^{\prime}(s)+\rho_{g} n(s)=1 \\
m(s) \rho_{g}+n^{\prime}(s)+k(s) \tau_{g}=0 \\
-n(s) \tau_{g}+k^{\prime}(s)=0
\end{array}\right.
$$


We will study the sytem of differential equations (40) for certain special cases as follows:

Case 1. If $m(s)=0$, the curve $\alpha$ is located in affine subspace combined with the vector space $S_{p}\left\{\eta_{2}(s), \eta_{3}(s)\right\}$ at the point $\alpha(s)$, then the system (40) turns into

$$
\left\{\begin{array}{l}
\rho_{g} n(s)=1, \\
n^{\prime}(s)+k(s) \tau_{g}=0, \\
-n(s) \tau_{g}+k^{\prime}(s)=0 .
\end{array}\right.
$$

From $(41)_{2}$ and $(41)_{3}$, we have

$$
k^{\prime \prime}(s)-\frac{\tau_{g}^{\prime}}{\tau_{g}} k^{\prime}(s)+\tau_{g}^{2} k(s)=0 .
$$

If we change the parameter as $t=\int_{0}^{s} \tau_{g} d s$, we obtain $\frac{d^{2} k}{d t^{2}}+k=0$. Thus the solution of $k$ is as follows:

$$
k=c_{1} \cos \left(\int_{0}^{s} \tau_{g} d s\right)+c_{2} \sin \left(\int_{0}^{s} \tau_{g} d s\right)
$$

where $c_{1}$ and $c_{2}$ are real constants. Also from $(41)_{1}$, it is clear that

$$
n(s)=\frac{1}{\rho_{g}}
$$

Rewriting (43) and (44) in (37), we obtain the curve $\alpha$ as

$$
\alpha(s)=\frac{1}{\rho_{g}} \eta_{2}(s)+\left[c_{1} \cos \left(\int_{0}^{s} \tau_{g} d s\right)+c_{2} \sin \left(\int_{0}^{s} \tau_{g} d s\right)\right] \eta_{3}(s) .
$$

Thereby, Blaschke vectors of the timelike ruled surface $X_{1}(s)$ are determined by the invariants of Darboux trihedron as follows:

$$
\begin{aligned}
& X_{1}(s)=\eta_{1}(s)+\xi\left[-\left[c_{1} \cos \left(\int_{0}^{s} \tau_{g} d s\right)+c_{2} \sin \left(\int_{0}^{s} \tau_{g} d s\right)\right] \eta_{2}(s)+\frac{1}{\rho_{g}} \eta_{3}(s)\right] \\
& X_{2}(s)=\eta_{2}(s)+\xi\left[-\left[c_{1} \cos \left(\int_{0}^{s} \tau_{g} d s\right)+c_{2} \sin \left(\int_{0}^{s} \tau_{g} d s\right)\right] \eta_{1}(s)\right] \\
& X_{3}(s)=\eta_{3}(s)+\xi\left[\frac{1}{\rho_{g}} \eta_{1}(s)\right] .
\end{aligned}
$$

Thus we can give the following result.

Corollary 9. The curve $\alpha$ is determined by (45) and the Blaschke vectors of the timelike ruled surface $X_{1}(s)$ are determined by the invariants of Darboux trihedron as in (46).

If $c_{1}=c_{2}=0$, the equation (46) turns

$$
\begin{aligned}
& X_{1}(s)=\eta_{1}(s)+\xi\left[\frac{1}{\rho_{g}} \eta_{3}(s)\right], \\
& X_{2}(s)=\eta_{2}(s), \\
& X_{3}(s)=\eta_{3}(s)+\xi\left[\frac{1}{\rho_{g}} \eta_{1}(s)\right] .
\end{aligned}
$$

Differentiating $(47)_{1}$ and $(47)_{3}$ with respect to $s$, and then using (9), we have

$$
p=\rho_{g}, p^{*}=\frac{\tau_{g}}{\rho_{g}}, q=-\tau_{g}, q^{*}=-1
$$

Corollary 10. The real and dual parts of the dual invariants of the timelike ruled surface $X_{1}$ are obtained as in (48). 
Case 2. If $n(s)=0$, the curve $\alpha$ is located in affine subspace combined with the vector space $S_{p}\left\{\eta_{1}(s), \eta_{3}(s)\right\}$ at the point $\alpha(s)$, then the system (40) turns into

$$
\left\{\begin{array}{l}
m^{\prime}(s)=1, \\
m(s) \rho_{g}+k(s) \tau_{g}=0, \\
k^{\prime}(s)=0 .
\end{array}\right.
$$

From $(49)_{1}$ and $(49)_{3}$, we have

$$
m=s+c_{1}, k=c_{2}
$$

and also from $(49)_{2}$, we obtain a ratio between the geodesic torsion and the geodesic curvature as

$$
\frac{\rho_{g}}{\tau_{g}}=-\frac{c_{2}}{s+c_{1}}
$$

Rewriting (50) in (37), we obtain the curve $\alpha$ as

$$
\alpha(s)=\left(s+c_{1}\right) \eta_{1}(s)+c_{2} \eta_{3}(s)
$$

Thereby, Blaschke vectors of the timelike ruled surface $X_{1}(s)$ are determined by the invariants of Darboux trihedron as follows:

$$
\begin{aligned}
& X_{1}(s)=\eta_{1}(s)+\xi\left[-c_{2} \eta_{2}(s)\right], \\
& X_{2}(s)=\eta_{2}(s)+\xi\left[-c_{2} \eta_{1}(s)+\left(s+c_{1}\right) \eta_{3}(s)\right], \\
& X_{3}(s)=\eta_{3}(s)+\xi\left[\left(s+c_{1}\right) \eta_{2}(s)\right] .
\end{aligned}
$$

Thus we can give the following result.

Corollary 11. The curve $\alpha$ is determined by (51) and the Blaschke vectors of timelike ruled surface $X_{1}(s)$ are determined by the invariants of Darboux trihedron as in (52).

If $c_{1}=c_{2}=0$, the equation (52) becomes

$$
\begin{aligned}
& X_{1}(s)=\eta_{1}(s) \\
& X_{2}(s)=\eta_{2}(s)+\xi\left(s \eta_{3}(s)\right), \\
& X_{3}(s)=\eta_{3}(s)+\xi\left(s \eta_{2}(s)\right) .
\end{aligned}
$$

Differentiating (53) 1 and (53) 3 with respect to $s$, and then using (9), we have

$$
p=\rho_{g}, p^{*}=0, q=-\tau_{g}, q^{*}=-1 .
$$

Thus we can give the following result.

Corollary 12. The real and dual parts of the dual invariants of the timelike ruled surface $X_{1}$ are obtained as in (54).

From (54) and (1), we get the following result.

Corollary 13. If $c_{1}=c_{2}=0$, then the timelike ruled surface generated by the line $X_{1}$ is a developable surface.

Case 3. If $k(s)=0$, the curve $\alpha$ is located in affine subspace combined with the vector space $S_{p}\left\{\eta_{1}(s), \eta_{2}(s)\right\}$ at the point $\alpha(s)$, then the system (40) turns into

$$
\left\{\begin{array}{l}
m^{\prime}(s)+\rho_{g} n(s)=1 \\
m(s) \rho_{g}+n^{\prime}(s)=0 \\
-n(s) \tau_{g}=0
\end{array}\right.
$$


From $(55)_{1}$ and $(55)_{3}$, we have

$$
m^{\prime \prime}(s)+\frac{\rho_{g}^{\prime}}{\rho_{g}}\left(1-m^{\prime}(s)\right)-\rho_{g}^{2} m(s)=0 .
$$

If we change the parameter as $t=\int_{0}^{s} \rho_{g} d s$ in (56), we obtain

$$
\frac{d^{2} m}{d t^{2}}-m=\frac{\rho_{g}^{\prime}}{\rho_{g}^{3}}
$$

Thus the solution of $m$ is as follows:

$$
m=c_{1} e^{\left(\int \rho_{0}^{s} \rho_{g} d s\right)}+c_{2} e^{\left(-\int \rho_{0}^{s} \rho_{g} d s\right)}+\frac{1}{D^{2}-1}\left(-\frac{\dot{\rho_{g}}}{\rho_{g}^{3}}\right),
$$

where $c_{1}$ and $c_{2}$ are real constants. Also from $(55)_{3}$, it is clear that

$$
n(s)=0
$$

Rewriting (57) and (58) in (37), we obtain the curve $\alpha$ as

$$
\alpha(s)=\left[c_{1} e^{\left(\int \rho_{g} \rho_{g} d s\right)}+c_{2} e^{\left(-\int_{0}^{s} \rho_{g} d s\right)}+\frac{1}{D^{2}-1}\left(-\frac{\dot{\rho_{g}}}{\rho_{g}^{3}}\right)\right] \eta_{1}(s),
$$

where $c_{1}, c_{2} \in R, \dot{\rho_{g}}=\frac{d \rho_{g}}{d t}, D=\frac{d}{d t}$.

Thereby, Blaschke vectors of the timelike ruled surface $X_{1}(s)$ are determined by the invariants of Darboux trihedron as follows:

$$
\begin{aligned}
& X_{1}(s)=\eta_{1}(s), \\
& X_{2}(s)=\eta_{2}(s)+\xi\left[c_{1} e^{\left(\int \rho_{0} d s\right)}+c_{2} e^{\left(-\int_{0}^{s} \rho_{g} d s\right)}+\frac{1}{D^{2}-1}\left(-\frac{\dot{\rho_{g}}}{\rho_{g}^{3}}\right)\right] \eta_{3}(s), \\
& X_{3}(s)=\eta_{3}(s)+\xi\left[c_{1} e^{\left(\iint_{0}^{s} \rho_{g} d s\right)}+c_{2} e^{\left(-\int_{0}^{s} \rho_{g} d s\right)}+\frac{1}{D^{2}-1}\left(-\frac{\dot{\rho_{g}}}{\rho_{g}^{3}}\right)\right] \eta_{2}(s) .
\end{aligned}
$$

Thus we can give the following result.

Corollary 14. The curve $\alpha$ is determined by (59) and the Blaschke vectors of timelike ruled surface $X_{1}(s)$ are determined by the invariants of Darboux trihedron as in (60).

If $c_{1}=c_{2}=0$, the equation (60) becomes

$$
\begin{aligned}
& X_{1}(s)=\eta_{1}(s) \\
& X_{2}(s)=\eta_{2}(s)+\xi\left[\frac{1}{D^{2}-1}\left(\frac{\rho_{g}^{\prime}}{\rho_{g}}\right)\right] \eta_{3}(s), \\
& X_{3}(s)=\eta_{3}(s)+\xi\left[\frac{1}{D^{2}-1}\left(\frac{\rho_{g}^{y}}{\rho_{g}}\right)\right] \eta_{2}(s) .
\end{aligned}
$$

Differentiating $(61)_{1}$ and $(61)_{3}$ with respect to $s$, and then using (9), we have

$$
p=\rho_{g}, p^{*}=0, q=\tau_{g}, q^{*}=0 .
$$


Thus we can give the following result.

Corollary 15. The real and dual parts of the dual invariants of the timelike ruled surface $X_{1}$ are obtained as in (62).

From (62) and (1), we get the following result.

Corollary 16. If $c_{1}=c_{2}=0$, then the timelike ruled surface generated by the line $X_{1}$ is a developable surface.

\section{Conclusion}

In this paper, the conditions between osculating strip curves and ruled surfaces were studied in dual Lorentz space $\mathbb{D}_{1}^{3}$. For this study, a system of differential equations characterizing both spacelike and timelike ruled surfaces were established in dual Lorentz space $\mathbb{D}_{1}^{3}$ by using the invariant quantities of osculating strip curves on the given ruled surfaces. Then the solutions of these systems were obtained for special cases. Regarding to these special solutions, some results of relations between osculating strip curves and ruled surfaces were given in dual Lorentz space $\mathbb{D}_{1}^{3}$.

\section{Competing interests}

The authors declare that they have no competing interests.

\section{Authors' Contributions}

All authors have contributed to all parts of the article. All authors read and approved the final manuscript

\section{References}

[1] N. Ayyıldı, A.C. Çöken, A. Yücesan, Differential-geometrical conditions between geodesic curves and ruled surfaces in the Lorentz space, Balk. J. Geo. Appl., 7(1) 2001, 1-12.

[2] N. Ayyıldız, A.C. Çöken, A. Kılıç, Differential-geometrical conditions between curves and semi-ruled surfaces in the semiEuclidean spaces, Tensor N. S., 62(2) 2000, 112-119.

[3] W. Blaschke, Vorlesungen über differential geometrie I, Ban I, Verlag Von Julius Springer-Verlag in Berlin, 1930.

[4] C. Ekici, E. Özüsağlam, On the method of determination of a developable timelike ruled surface, KJSE- Kuwait Journal of Science \& Engineering, 39(1A) 2012, 19-41.

[5] C. Ekici, A.C. Çöken, The integral invariants of parallel timelike ruled surfaces, JMAA- Journal of Mathematical Analysis and Applications, 393(1) 2012, 97-107.

[6] H.W. Guggenheimer, Differential geometry, Mc. Graw-Hill Book Company, New York, 1963.

[7] Ş. Nizamoğlu, N. Gülpınar, Differential-geometrical conditions between curves and ruled surfaces, J. Fac. Scie. Ege Uni. 16(1) 1993, 53-62.

[8] B. O 'Neill, Semi-Riemannian geometry with applications to relativity, Academic press Inc, London, 1983.

[9] Ö. Pasinli, Ruled surfaces, Master Thesis, Grad. Sch. Nat. Appl. Sci. Dokuz Eylül Uni., İzmir, 1997.

[10] Ü, Pekmen, Differential-geometrical conditions between geodesic curves and ruled surfaces, J. Fac. Scie. Ege Uni., 16(1), 1995, 67-74.

[11] E. Study, Die geometrie der dynamen, Verlag Teubner, Leipzig, 1933.

[12] M. Şişman, Differential geometrical conditions between curvature and osculating strip curves and ruled surfaces, Master Thesis, Grad. Sch. Nat. Appl. Sci. Dokuz Eylül Uni., İzmir, 1995.

[13] Tutar A. IL $L^{3}$ Lorentz uzayında küresel ĕgriler ve Joachimsthal teoremi, Ph D Dissertation, Ondokuz Mayıs Üniversitesi, 1994, 36-37 (in Turkish).

[14] H.H. Uğurlu, A. Çalışkan, Darboux ani dönme vektörleri ile spacelike ve timelike yüzeyler geometrisi, CBÜ Yay., Manisa, 2012.

[15] G.R. Veldkamp, On the use of dual numbers, vectors and matrices in instantaneous spatial kinematics, Mech. Math. Theory, 11, 1976, 141-156. 